LIAMES 10 - pp. 101-113, Primavera 2010

\author{
Antônia Alves Pereira ${ }^{1}$ \\ (Universidade Federal do Pará)
}

\title{
A nominalização e as orações relativas no Asurini do Xingu (Tupi-Guarani) ${ }^{2}$
}

\begin{abstract}
In this work we present the process of nominalization in Asurini of Xingu and its role in the formation of relative clauses. We show that this language does not have relative pronouns, relativization being performed by a set of nominalizers. These nominalizers are selected in accordance with the function of the nuclear noun in the relative clause. The resources used in the coding of elements in this type of clause vary according to the nature of the nominalizer, as well as according to the function of the nuclear noun in the clause.
\end{abstract}

KEYWORDS: Nominalization; Relative clauses; Asurini language.

RESUMO: Neste trabalho, apresentamos o processo de nominalização no Asurini do Xingu e seu papel na formação das orações relativas. Mostramos que nessa língua não há pronomes relativos, sendo a relativização formada por um conjunto de nominalizadores. Esses nominalizadores são selecionados conforme a função do nome núcleo na oração relativa. Os recursos utilizados na codificação dos elementos nesse tipo de oração variam de acordo com a natureza do nominalizador e da função do nome núcleo da oração relativa.

PALAVRAS-CHAVE: Nominalização; Cláusulas relativas; Língua asurini do Xingu.

\section{INTRODUÇÃO}

Este trabalho tem como objetivo analisar o processo de nominalização no Asurini do Xingu e seu papel na formação das orações relativas. Essa língua, conforme classificação de Rodrigues (1986), faz parte da família Tupi-Guarani, tronco Tupi. O povo asurini do Xingu vive no município de Altamira, estado do Pará, e sua população é composta por aproximadamente 150 pessoas.

${ }^{1}$ Professora do quadro permanente da Universidade Federal do Pará (UFPA).

${ }^{2}$ Este artigo é parte do capítulo 6 de minha Tese de Doutorado intitulada "Estudo Morfossintático do Asurini do Xingu", orientada pela prof ${ }^{a} \operatorname{Dr}^{a}$ Lucy Seki, defendida no Instituto de Estudos da Linguagem, Universidade Estadual de Campinas em 2009. A pesquisa de campo contou com o apoio financeiro do CNPq através da concessão de taxa de bancada. 
No decorrer desse trabalho, procuramos mostrar que existe uma estreita relação entre a nominalização na derivação deverbal e a nominalização como estratégia de relativização no que se refere aos morfemas nominalizadores.

Este artigo está dividido em três partes. Na primeira, tratamos da nominalização deverbal, apresentando a descrição dos nominalizadores, na segunda apresentamos as estratégias de relativização e as posições e funções relativizáveis na língua e na última parte apresentamos nossas conclusões.

\section{A NOMINALIZAÇÃo}

A língua Asurini do Xingu, assim como outras línguas da família Tupi-Guarani, apresenta as nominalizações como um forte recurso gramatical para a formação de SNs (Sintagmas Nominais). Após a nominalização, tem-se um SN que desempenha as funções sintáticas de um nome na oração independente: sujeito $(\mathbf{S a}, \mathbf{S o} \text { e } \mathbf{A})^{3}$ e a de objeto $(\mathbf{O})$. Até esse estágio de nossa pesquisa, constatamos, nessa língua, os seguintes nominalizadores: $\{$-tat $\},\{$-tap $\},\{$-emi $\},\{$-ipyt $\},\{$-ama'e $\},\{$-ima'e $\}$ e $\{$-wat $\}$. Esses nominalizadores afixam-se à raiz verbal, fazendo com que a oração se nominalize e passe a desempenhar uma função sintática em relação à oração principal, isto é, o que antes era um predicado verbal deixa de exercer funções verbais, passando a ser uma estrutura nominalizada e sintaticamente dependente, exercendo funções típicas de um nominal, podendo, inclusive, receber toda morfologia que um nominal recebe.

Todos os nominalizadores acima citados, com exceção de $\{$-ama'e $\},\{$-ima'e $\}$, após se afixarem ao radical verbal, recebem o morfema $\{-\mathbf{a}\}$. Esse morfema é recorrente nas línguas da família Tupi-Guarani. Em muitas delas ainda marca todas as funções tipicamente nominais. No Asurini do Xingu, dá indícios de ter marcado as funções nominais em estágios anteriores da língua. No estágio atual, parece está perdendo o status gramatical em alguns contextos (para mais informações sobre esse morfema, cf. Pereira 2009). Esse morfema pode aparecer imediatamente depois dos nominalizadores ou depois do morfema $\{$-et $\}$, marcador de tempo passado nominal (mais informações sobre o morfema \{-et\} encontram-se em Pereira (2005).

Os afixos nominalizadores do Asurini do Xingu, em virtude do processo de alomorfia, podem adquirir outras formas além das apresentadas acima. Os fenômenos mais comuns são a queda da consoante coronal inicial e as mudanças de $\mathbf{t}$ para $\mathbf{v}$ e $\mathbf{p}$ para $\mathbf{r}$ na posição final do morfema quando estes últimos encontram-se antes do morfema $\{-\mathbf{a}\}$.

Passamos, a seguir, à descrição dos nominalizadores.

\subsection{Nominalizador de agente}

O nominalizador $\{$-tat $\}$ ocorre com radicais verbais transitivos. Após a ocorrência desse nominalizador, o nome derivado adquire traço semântico [+agentivo]. Pelo que podemos perceber até esse momento da pesquisa, a nominalização do radical verbal com esse nominalizador não afeta sua valência.

\footnotetext{
${ }^{3}$ S, A, O, Sa e So são utilizados aqui tal como em Dixon (1994).
} 
PEReira: A nOMinALiZAÇão E AS ORAÇÕES RELATIVAS No Asurini...

(1)

$\begin{array}{lll}\text { ga } & \text { bola } & \text { mabak-ar-a } \\ \text { 3sg.Mas } & \text { bola } & \text { jogar-Nom-N } \\ \text { 'ele é jogador de bola' } & \end{array}$

\subsection{Nominalizador de ação/estado}

O nominalizador $\{$-tap\} ocorre com radicais verbais transitivos e intransitivos formando nomes de ação, estado conforme se pode conferir nos exemplos (2), (3) e (4) abaixo.

mej

maritykwat

'mentir'

mej-tap-a

'ação de mentir'

(3)

(4) akup 'trabalhar'

'quente' maritykwat-tap-a

akup-tap-a 'ação de trabalhar'

'estado febril'

\subsection{Nominalizador de nome paciente/objeto}

A nominalização de paciente se dá com o morfema $\{-\mathbf{e m i}\}$, cuja ocorrência é restrita a radicais transitivos. Após a nominalização com esse morfema, o nome derivado é sempre precedido pelo relacional $\{\mathbf{r}-\}$, evidenciando a existência de um termo dependente antes dele.

(5) dje r-emi-u'-er-a

1sg Rel-Nom-comer-Pas-N

'o que eu comi/ o que foi comido por mim'

(6) ene r-emi-avyki- $\varnothing$

2sg Rel-Nom-bater-N

'o que você bateu'

\subsection{Nominalizador de nome paciente}

O nominalizador $\{$-ipyt $\}$, analogamente ao $\{-\mathbf{e m i}\}$, anexa-se apenas a radicais transitivos. Sua ocorrência se dá sempre com o morfema marcador de tempo passado nominal $\{$-et\}. Semanticamente, o nome derivado adquire o traço [+paciente].

(7) i-djuka-pyr- er-a

3-matar-Nom-Pas-N

'o que foi morto'

(8) i-manaka-pyr- er-a

3-morder-Nom-Pas-N

'o que foi mordido' 


\subsection{Nominalizador atributivo}

O nominalizador \{-ama'e\}, que se realiza como \{-ma'e\} depois de vogal, só ocorre com radicais intransitivos ativos ou descritivos. Esse nominalizador, conforme o nome já sugere, semanticamente atribui ao sujeito características expressas pelo verbo. Nesse sentido, ele é análogo ao morfema \{-ama'e\} do Kamaiurá, que segundo Seki (2000:122), deriva nominais que indicam, respectivamente, 'o que realiza X', 'o que é $X$ ' e o 'que tem $X^{\prime}$. A seguir, exemplos do Asurini do Xingu.

(9) u-manu-ma'e

3-morrer-Nom

'o que está morto'

(10) u-furahaj-ma'e

3-dançar-Nom

'o que dança'

(11) i- akuv -ama'e

3-febril -Nom

'o que está quente, febril'

(12) i-ajver-ama'e

3-feio-Nom

'o que é feio'

\subsection{Nominalizador atributivo negativo}

O nominalizador $\{$-ima'e $\}$ é a contraparte negativa de $\{$-ama'e\}, ou seja, ele atribui negativamente ao sujeito a característica expressa pelo radical verbal. Do mesmo modo que $\{$-ama'e $\}$ acrescenta-se a verbo intransitivo e descritivo.

(13) u-manu-ima'e

3-morrer-Nom

'o que não está morto'

(14) kudjema'e u-furaj-ima'e

homem 3-dançar-Atr.negativo

'o homem que não dança'

(15) i-katu-ima'e

3-ser. bom-Nom

'o que não está bom, não presta'

(16) djupypygy i-katu-ima'e

arroz 3-bom/bonito- nom

'o arroz está estragado' 
Tanto o nominalizador $\{$-ama'e $\} \sim\{$ ma'e $\}$ quanto o $\{$-ima'e $\}$ diferenciam-se dos demais nominalizadores da língua pelo fato de as raízes nominalizadas com eles continuarem funcionando com elementos pronominais dos verbos independentes.

\subsection{Nominalizador de circunstância}

O nominalizador \{wat\} se anexa a elementos que desempenham funções adverbiais, formando, a partir daí, nominais. Em todos os registros que temos, esse nominalizador é sempre seguido pelo sufixo $\{-\mathbf{a}\}$.

$$
\begin{aligned}
& \text { ka-ve war-a } \\
& \text { roça-Loc Nom-N } \\
& \text { 'o que é da roça' }
\end{aligned}
$$

(18) kare war-a

hoje Nom-N

'o que é de hoje'

$$
\begin{aligned}
& \text { y'-ve war-a } \\
& \text { água-Loc Nom-N } \\
& \text { 'o que é da água' }
\end{aligned}
$$

\begin{tabular}{|c|c|c|c|c|c|}
\hline & Agente & Paciente & $\begin{array}{l}\text { Atributivo } \\
\text { Positivo }\end{array}$ & $\begin{array}{l}\text { Atributivo } \\
\text { Negativo }\end{array}$ & $\begin{array}{l}\text { Ação/Estado } \\
\text { Oblíquo }\end{array}$ \\
\hline V.T & $\{$-tat $\}$ & $\begin{array}{l}\{- \text { emi- }\} \\
\{-i p y t\}\end{array}$ & & & \\
\hline V.T e V. I. & & & & & $\{$-tap $\}$ \\
\hline V.I e Descritivo & & & $\begin{array}{l}\{- \text { ma'e }\} \sim \\
\{\text {-ama'e }\}\end{array}$ & $\{$-ima'e\} & \\
\hline
\end{tabular}

Na seqüência, apresentamos um quadro com os nominalizadores do Asurini do Xingu:

Tabela 1: Nominalizadores do Asurini do Xingu

\section{AS NOMINALIZAÇÕES E AS ORAÇÕES RELATIVAS NOASURINI DO XINGU}

No Asurini do Xingu, assim como em outras línguas da família Tupi-Guarani, não há pronomes relativos, ao contrário de línguas como a Portuguesa e a Inglesa que usam esses pronomes na construção de cláusulas relativas. O Asurini do Xingu usa como estratégia de formação das orações relativas as estruturas nominalizadas descritas até aqui. Vale ressaltar que as nominalizações, nessa língua, ocorrem também em outros tipos de orações dependentes que não serão tratadas aqui. 
Para o tratamento das orações relativas aqui, adotamos a conceituação de Comrie (1981:136) "A relative clause then consists necessarily of a head and a restricting clause. The head in itself has a potential range of referents, but the restricting clause restricts this set by giving a proposition that must be true of the actual referents of the over-all construction". Como em outras línguas, as relativas do Asurini, modificam um nome núcleo da oração principal.

As orações relativas se distinguem das orações principais, nas línguas do mundo, por vários meios. A seguir, apresentamos dois aspectos que, conforme Keenan (1985), distinguem esses dois tipos de orações em diferentes línguas:

- as orações relativas do tipo pós-nominal podem ser precedidas por complementalizador morfologicamente invariante; analogamente, as relativas pré-nominais podem ser seguidas por uma forma morfologicamente invariante.

- o verbo da oração relativa apresenta redução na morfologia, como a marcação de tempo-aspecto e concordância em relação a verbos de oração declarativa.

Esta última é a estratégia utilizada pelo Asurini do Xingu.

Keenan classifica as orações relativas de núcleo externo como relativa de núcleo externo pós-nominal e relativa de núcleo externo pré-nominal. Conforme esse autor:

\begin{abstract}
"We shall base our classification of RCS on those which do present a domain noun, leaving it to the field worker to notice cases (...) in which the domain noun is absent: and for each type of RC we distinguish, we shall discuss the positions in which determiners may be presented.

(...) the domain noun (...) occurs outside the Srel. We shall refer to such RCS as external or headed relatives. Since Srel occurs to the right of the domain noun (...) we shall refer to them more specifically as postnominal external relatives. Correspondingly, RCS in which the domain noun is outside of Srel and the latter occurs to the latter left of the domain noun will be called prenominal external relatives" (Keenan 1986:143)
\end{abstract}

Neste trabalho, seguimos essa análise de Keenan. Em Asurini, há orações relativas com núcleo externo pré-nominal e núcleo externo pós-nominal. Em nossos dados até o momento, o tipo pós-nominal é o mais recorrente, sendo menos comum o tipo pré-nominal. A predominância do tipo pós-nominal na língua, que tem como ordem predominante SOV, não está de acordo com a tendência universal observada por Keenan (1985:144): "More specifically, postnominal RCS are almost the only type attested in verb-initial languages", Línguas de ordem SOV, caso do Asurini do Xingu, tendem a ter como tipo predominante a relativa pré-nominal. Outro fator que também contraria a tendência universal é o fato de a língua apresentar relativas pós-nominais e relativas pré-nominais, já que segundo Keenan (1985) não é tão comum as línguas apresentarem esses dois tipos de relativas ao mesmo tempo, e no caso de apresentarem, é mais comum aparecer no tipo de língua que tem como ordem predominante $\mathrm{SVO}^{4}$.

As orações relativas pré-nominais distinguem-se das relativas pós-nominais em algumas línguas, segundo Keenan (1985:160): "A more regular diference between prenominal and postnominal RCS concerns the form of the main verbo of Srel...". Em Asurini do Xingu, as relativas pré-nominais e as pós-nominais são morfologicamente idênticas.

\footnotetext{
${ }^{4}$ Apesar de a ordem predominante no Asurini do Xingu ser SOV, a ordem SVO é encontrada na língua.
} 


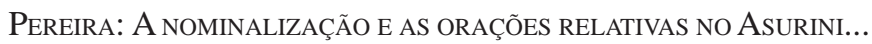

a) Relativa de núcleo externo pós-nominal

myra mani'aka u-kytyk [ene r-emi-pepin- $\varnothing$ ]

Npr mandioca 3-ralar 2sg Rel-Nom-descascar-N

'Myra ralou a mandioca que você descascou'

Lit: /Myra ralou a mandioca descascada por você/

(21)
tadjau
Ø-tx̃
[apevu
r-emi-mumuku- $\varnothing]$
u-jũn
porco
Rel-focinho Npr
Rel-NZ-furar -N
3-inchar

'o focinho do porco que Apevu furou inchou'

b) Relativa de núcleo externo pré-nominal
[kujĩ
py'yk-ar-er-a]
kudjema'e
aha
mulher
pegar-Nom-Pas-N
homem
ir
'o homem que pegou a mulher fugiu'

Conforme ilustram os exemplos acima, não existe distinção morfológica entre a relativa de núcleo externo pré-nominal e a relativa de núcleo externo pós-nominal. A distinção dá-se através da sintaxe da língua, isto é, através da posição em que ocorre o núcleo: à direita ou à esquerda do Srel.

Como já mencionado, a cláusula relativa tem como função modificar um nominal da oração principal, o qual pode estar desempenhando as funções: $\mathrm{O}, \mathrm{A}, \mathrm{S}$ e Obl.

Em relação aos elementos que funcionam como sujeito nas orações relativas em Asurini do Xingu, a codificação é feita por nominais e pronomes pessoais (acompanhados por relacionais), prefixo relacional de terceira pessoa especificada e prefixos da série I em alguns casos. Abaixo, o quadro II apresenta a série de pronomes pessoais e a série I de prefixos ativos.

Tabela 2: Pronomes pessoais e série I dos prefixos ativos

\begin{tabular}{|c|c|c|}
\hline Pessoas & Pronomes pessoais & Prefixos da série I \\
\hline $1^{\mathrm{a}}$ p.sg & dje & a- \\
\hline $2^{\mathrm{a}}$ p. sg & ene & ere- \\
\hline $1^{\mathrm{a}}$ p. incl. & djane & txa- \\
\hline $1^{\mathrm{a}} \mathrm{p} . \mathrm{excl}$ & ure & uru- \\
\hline $2^{\mathrm{a}} \mathrm{p} \mathrm{Pl}$ & pene & pe- \\
\hline $3^{a}$ p. sg.Mas & ga & \multirow{3}{*}{$\mathrm{u}-$} \\
\hline $3^{\mathrm{a}}$.p.sg. Fem & $\tilde{\mathrm{e}}$ & \\
\hline $3^{\mathrm{a}} \cdot \mathrm{p} . \mathrm{Pl}$ & gy & \\
\hline
\end{tabular}


A seguir exemplos ilustrativos:

a) Sa codificado por prefixo da série I

(23a) u-ur-ama'e u-djau

3-vir- Nom 3-banhar

'o que veio banhou-se'

(23b) u-manu-ima'e

3-morrer-Nom

'o que não está morto'

b) So codificado por relacional i- (terceira pessoa especificada)

(24) i-marin-ama'e u-manu

3-estar doente-Nom 3-morrer

'o que estava doente morreu'

c) A codificado por pronome pessoal

$\begin{array}{lllll}\text { (25) } & \text { a-etxak } & \text { kuj̃ } & \text { ene u'i } & \text { mana-av-er-a } \\ \text { 1-ver } & \text { mulher } & \text { 2sg farinha } & \text { dar-Nom-Pas-N }\end{array}$

'eu vi a mulher a quem você deu farinha'

c) A codificado por nominal
kwa'I u-djuka
maja kunumi
mamak-ar-er-a
Npr 3-matar
cobra menino
morder-Nom-Pas-N
'Kwa'I matou a cobra que mordeu o menino'

Conforme se pode perceber através dos exemplos acima, há uma sensível diferença entre os nominalizadores $\{$-ma'e $\}$ e $\{$-ima'e $\}$ em relação aos demais nominalizadores da língua. Com o acréscimo desses dois nominalizadores há um radical verbal, a estrutura nominalizada continua funcionando com prefixo ativo da série I.

Os exemplos acima também mostram que não existe um critério morfológico que faça distinção entre sujeito e objeto nessas orações. Tampouco existe distinção formal entre os diferentes tipos de sujeitos, como existe nas orações independentes. A sintaxe da língua é de fundamental importância para fazer tal distinção, pois é através dela que se sabe o tipo de argumento que atua em uma dada posição: a valência do verbo e o nominalizador fazem com o que o falante identifique os argumentos, interpretando-os como $\mathrm{Sa}$, So, $\mathrm{A}$ ou $\mathrm{O}$, independentemente da codificação dos mesmos.

A seguir, trataremos das posições e funções relativizáveis nas orações. 


\subsection{Posições relativizáveis na cláusula em Asurini do Xingu}

As posições relativizáveis nas cláusulas em Asurini do Xingu são S, A, O, Oi/Oblíquos, e encontram-se em conformidade com a hierarquia de acessibilidade de Keenan e Comrie (1977). Abaixo, reproduzimos a hierarquia desses autores:

\section{$\mathrm{SU}>\mathrm{DO}>\mathrm{OI}>\mathrm{OBL}>\mathrm{GEN}>\mathrm{O}$ comp}

Conforme podemos observar através da hierarquia acima, a posição de sujeito é a mais relativizável nas línguas. Assim, se uma língua permite relativização, a posição de sujeito é uma das posições relativizadas. Apesar de todos os sujeitos poderem ser relativizados em uma dada língua, é possível que nessa língua existam sujeitos menos relativizáveis que outros, os intransitivos, por exemplo, são mais relativizáveis que os transitivos. Assim há línguas que permitem relativização de sujeito intransitivo, mas não permite a relativização de sujeitos transitivos (Keenan 1985).

Percebemos até esse estágio da nossa pesquisa com o Asurini do Xingu, que essa língua relativiza as posições que vão de sujeito a Obliquo na hierarquia apresentada acima. A posição sujeito é a mais propensa à relativização no Asurini do Xingu, os sujeitos intransitivos são mais propensos à relativização que os sujeitos de verbos transitivos, segundo nossa análise.

Na sequência, vamos verificar as posições e funções relativizáveis no Asurini do Xingu.

\subsubsection{Relativização de $S$}

Em Asurini do Xingu Sa e So ocorrem em construções que podem ser nominalizadas com os seguintes afixos: $\{$-ma'e $\} \sim\{$-ama'e $\},\{$-ima'e $\}$ e $\{$-ipyt $\}$. Os dois primeiros nominalizadores se anexam a radicais verbais intransitivos e descritivos, como podemos ver nos exemplos que seguem. Ressaltamos que esses nominalizadores são usados exclusivamente com terceira pessoa.

$$
\begin{aligned}
& \text { a-etxak kuj̃ u-furu }{ }^{5} \text {-djuaka-ma'e } \\
& \text { 1-ver mulher 3-Gn-pintar-Nom } \\
& \text { 'eu vi a mulher que pinta (gente)' }
\end{aligned}
$$

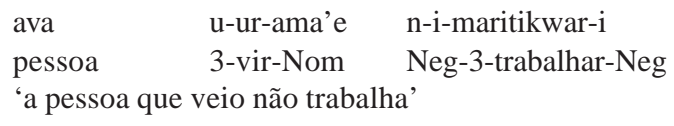

Quando o verbo da oração relativa é intransitivo ativo, o sujeito é codificado pelo prefixo $\{-\mathbf{u}\}$ e quando intransitivo descritivo é codificado pelo prefixo $\{-\mathbf{i}\}$. A marcação de pessoa diferente para o sujeito dessas orações mostra, pois, a natureza variada dos verbos intransitivos.

${ }^{5}$ Em Asurini do Xingu, a incorporação de um objeto genérico ao verbo torna-o intransitivo. O verbo djuaka, por exemplo, sem o morfema $\{$-furu $\}$ seria transitivo. 
(28) u-ur-ama'e u-djauk

3-vir- Nom 3-banhar

'o que veio banhou-se'

(29) i-marin-ama'e u-manu

3-estar doente-Nom 3-morrer

'o que estava doente morreu'

O morfema \{-ipyt \}, no Asurini do Xingu, tem função análoga àquela apontada por Seki (2000) para o Kamaiurá: a de desagentivizador que, ao anexar-se a radicais verbais transitivos, acarreta mudança nas relações sintáticas, pois força o argumento $\mathbf{O}$ a desempenhar a função So, ou seja, um argumento que desempenhava a função de objeto passa a desempenhar a função de sujeito.

$\begin{array}{lcc}\text { u-manu } & \text { kunumI } & \text { i-manaka-pyr-er-a } \\ \text { 3-morreu } & \text { menino } & \text { 3-estar cortado-Nom-Pas-N } \\ \text { 'morreu o menino que estava cortado' }\end{array}$

\subsubsection{Relativização de A}

O argumento A da oração relativa se relativiza com o morfema $\{$-tat $\}$, que, conforme já tratamos, tem traço semântico \{+agentivo\}. O argumento relativizado, isto é, o núcleo tem a função de A na oração relativa. O elemento genitivizado junto ao verbo nominalizado é interpretado como $\mathrm{O}$.

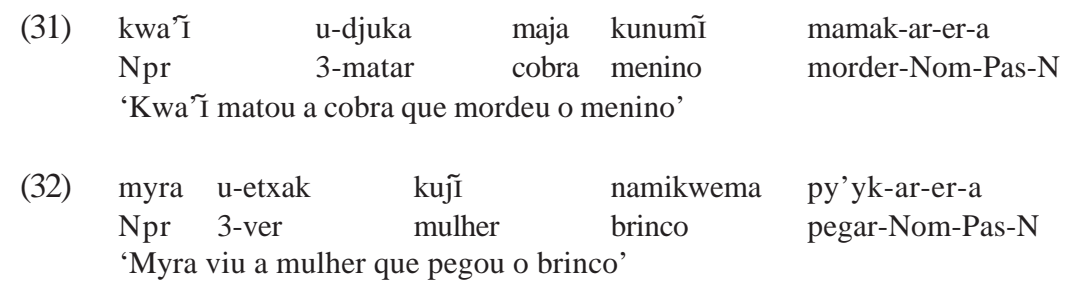

\subsubsection{Relativização de $O$}

Quando o nome núcleo tem na oração relativa a função de $\mathrm{O}$, o nominalizador é $\{-\mathbf{e m i}\}$. O elemento genitivizado junto ao verbo nominalizado é interpretado como A. O morfema $\{$-emi $\}$ se comporta morfologicamente como um nome pertencente à classe $\{\mathbf{r}-\}$ dos nomes, isto é, recebe os mesmos prefixos relacionais que um nome da classe $\{\mathbf{r}-\}$ recebe (mais informações sobre esse prefixo se encontram em Pereira 2009, cap. 3).

$$
\begin{array}{lllll}
\text { myra mani'aka } & \text { u-kytyk } & \text { ene } & \text { r-emi-pepI- } \varnothing \\
\mathrm{Npr} \text { mandioca } & \text { 3-ralar } & \text { 2sg } & \text { Rel-Nom-descascar-N } \\
\text { 'Myra ralou a mandioca que tu descascaste' } &
\end{array}
$$


Pereira: A nOminAlizaÇão E AS ORAÇÕES RElativas No ASURINI...

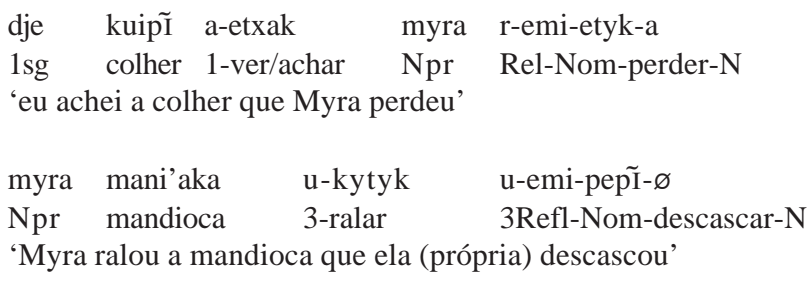

Os exemplos (33) e (34) mostram o prefixo $\{\mathbf{e m i} \mathbf{-}\}$ junto a sujeitos codificados por segunda pessoa e por nominal, respectivamente, recebendo, pois o prefixo $\{\mathbf{r}-\}$. O exemplo (35) mostra o prefixo \{emi- $\}$ junto a um sujeito que é co-referente ao sujeito da oração principal, recebendo, pois, o prefixo reflexivo de terceira pessoa $\{\mathbf{u}-\}$.

\subsubsection{Relativização de oblíquo}

A estratégia utilizada pelo Asurini do Xingu para a relativização de oblíquo é a sufixação do morfema $\{$-tap\} à raiz verbal da oração dependente, como podemos observar nos dados abaixo:

$$
\begin{array}{ccccc}
\text { a-etxak } & \text { kujema'e } & \text { kuj̃ } & \text { t-yru } & \text { futuka-av-er-a } \\
\text { 1-ver } & \text { homem } & \text { mulher } & \text { 3-roupa } & \text { lavar-Nom-Pas- } \\
\text { 'eu vi o homem para quem a mulher lava roupa' } &
\end{array}
$$

1-ver homem mulher 3-roupa lavar-Nom-Pas-N

$\begin{array}{lllll}\text { a-etxak } & \text { kuj̃ } & \text { ene } & \text { u'i } & \text { mana-av-er-a } \\ \text { 1-ver } & \text { mulher } & \text { 2sg } & \text { farinha } & \text { dar-Nom-Pas-N }\end{array}$

'eu vi a mulher a quem você deu farinha'

Os dados mostram que após a afixação do morfema \{-tap\} à raiz do verbo transitivo da oração dependente, o nome núcleo desempenha a função de OI na oração relativa.

\section{CONCLUSÃO}

Nesse trabalho, mostramos que o recurso utilizado pelo Asurini do Xingu como estratégia de relativização é a nominalização do verbo da oração dependente. A nominalização é obtida através de um conjunto de morfemas que atua em radicais verbais transitivos e intransitivos.

No decorrer do trabalho mostramos que existe uma estreita relação morfológica entre a nominalização deverbal e a nominalização como estratégia de relativização. Mostramos também que as escolhas dos argumentos na cláusula relativa são guiados pelos nominalizadores que atuam nessas cláusulas.

A nominalização no Asurini do Xingu apresenta vários aspectos em comum com outras línguas da mesma família, dentre essas línguas, citamos aqui o Kamaiurá. Os morfemas nominalizadores são idênticos morfologicamente e partilham de vários traços sintáticos, da mesma forma que são idênticas algumas regras de alomorfia nas duas línguas. 
Brandon e Seki (1981) demonstram que a nominalização enquanto recurso de subordinação é muito comum às línguas indígenas do tronco Tupi.

Por fim, esperamos que o trabalho possa contribuir para estudos tipológicos e/ou histórico-comparativos envolvendo línguas da família tupi-guarani e do tronco tupi.

\section{REFERÊNCIAS BIBLIOGRÁFICAS}

BRANDON, Frank R.; SEKI, Lucy (1981). Interrogativos e complementizadores em línguas Tupi. Estudos Lingüísticos 5: 107-114.

COMRIE, Bernard (1981). Language Universals and Linguistic Typology. Oxford: Basil Blackwell.

DIXON, R. M. W. (1994). Ergativity. Cambridge: Cambridge University Press.

KEENAN, Edward L. (1985). Relatives clauses. In Timothy Shopen (ed.).Language typology and syntactic description. Complex constructions, vol 2: 141-170. Cambridge: Cambridge University Press.

; COMRIE, Bernard (1977). NP accessibility and universal grammar. Linguistic inquiry 8(1): 66100.

PEREIRA, Antônia Alves (2009). Estudo Morfossintático do Asurini do Xingu. Tese de Doutorado em Linguística. Campinas: Universidade Estadual de Campinas (UNICAMP)

.(2005). Aspectos morfológicos da língua Asurini do Xingu. Dissertação de Mestrado em Letras. Belém, Pará: Universidade Federal do Pará (UFPA).

RODRIGUES, Aryon Dall'Igna (1986). Línguas Brasileiras. Para o conhecimento das línguas indígenas. São Paulo: Edições Loyola.

SEKI, Lucy (2000). Gramática do Kamaiurá: Língua tupí-guaraní do alto Xingu. Campinas: Editora da UNICAMP/Imprensa Oficial.

\section{Abreviaturas}

A Sujeito de verbo transitivo

Atr Atributivo

Fem Falante de sexo feminino

Fut Tempo futuro

Gn Morfema genérico

Loc Locativo

Mas Falante de sexo masculino

Nom Nominalizador

N Nominalizador de argumento nuclear

Neg Negação

$\mathrm{Npr} \quad$ Nome próprio

$\mathrm{O}$ Objeto direto

Obl Oblíquo

Orel Oração relativa

Pas Tempo passado 
Pereira: A nominalização E AS ORAÇões Relativas no Asurini...

$\begin{array}{ll}\text { Part } & \text { Partícula } \\ \text { Pl } & \text { Plural } \\ \text { Posp } & \text { Posposição } \\ \text { Refl } & \text { Reflexivo } \\ \text { Rel } & \text { Prefixo relacional } \\ \text { S } & \text { Sujeito de verbo transitivo } \\ \text { Sa } & \text { Sujeito de verbo transitivo ativo } \\ \text { So } & \text { Sujeito de verbo transitivo descritivo } \\ \text { SN } & \text { Sintagma nominal } \\ 1 & \text { Primeira pessoa } \\ 2 & \text { Segunda pessoa } \\ 3 & \text { Terceira pessoa }\end{array}$

Recebido: 27/4/2010

Versão revista (1): 30/7/2010

Versão revista (2): 14/9/2010

Aceito: 25/11/2010. 